\title{
Socioeconomic circumstances and the risk of bowel cancer in Northern Ireland
}

\author{
F Kee, R Wilson, S Currie, J Sloan, R Houston, B Rowlands, J Moorehead
}

Department of

Epidemiology and

Public Health,

Queens University,

Belfast

F Kee

Northern Ireland

Centre for Clinical

Oncology, Belfast

R Wilson

R Houston

Information and IT

Department, Northern

Health and Social

Services Board,

Ballymena

$S$ Currie

Department of

Pathology,

Queens University,

Belfast

J Sloan

Department of

Surgery,

Queens University,

Belfast

B Rowlands

Department of Surgery, Ulster, North Down and Ards

Hospital Trust

J Moorehead

Correspondence to:

Dr F Kee, Department of

Public Health Medicine,

Northern Health and Social

Northern Health and Socia

Services Board, County
Hall, Ballymena BT 42 QB

Accepted for publication

May 1996

\begin{abstract}
Objective - To describe the variation in the incidence of colorectal cancer across Northern Ireland and relate it to factors associated with community deprivation.

Design - This was a cross sectional descriptive study.

Setting - Incidence data were obtained from a population based register for the period 1990-91. Small areas were characterised by their "affluence", or lack of it, by deriving a Townsend deprivation score for each electoral ward, using information from the 1991 census.

Participants, main outcome measures, and statistical methods - The age standardised incidence was calculated for all colorectal cancer cases diagnosed histologically in 1990-91. Electoral wards were grouped into quintiles of the population after ranking of their Townsend scores and the association with incidence was studied using Poisson regression.
\end{abstract}

Results - The age standardised colorectal cancer incidence ranged from 22.5 (for quintile 1) to $29.9 / 100000$ (quintile 5) for men but the trend for women was less regular and rates were $18.4,23.8,27.3$, 26.5 , and $23.9 / 100000$ for quintiles $1-5$ respectively (that is, from the most "affluent" to the most "deprived" fifths of the population). After adjusting for age and sex in Poisson regression, there was a significant association between the total colorectal cancer incidence and levels of community deprivation. The rate ratio for the most deprived quintile of the population (compared with the least) was 1.28 $(95 \%$ CI 1.06,1.53). The effect was stronger for rectal cancer than for colonic cancer. There was no association between community deprivation and the cancer stage at diagnosis.

Conclusions - In this population, the colorectal cancer incidence is associated with the level of material deprivation. The disease stages at the time of diagnosis in patients from more deprived areas seem to be comparable with those of patients from affluent areas. As others have shown, associations such as these are not explicable entirely on the basis of the distribution of known risk factors. Further research is needed to determine plausible mechanisms for the association.

(f Epidemiol Community Health 1996;50:640-644)
Cancers of the colon and rectum show substantial international variation in the incidence. Rates in men range from $0.7 / 100000$ in The Gambia to $35.9 / 100000$ in Connecticut for colon cancer and from $0.7 / 100000$ in The Gambia to $22.9 / 100000$ in Moravia for rectal cancer. ${ }^{1}$ Even though disease that is apparently "sporadic" may have a considerable genetic component, the evidence from studies of migrant populations suggests that much of the cross cultural variation in incidence may be attributable to environmental factors, such as diet. On the other hand, the variation in the incidence within a country is more modest. The age standardised registration ratios within England and Wales range from 0.86 to 1.26 (in London and Montgomeryshire, respectively). ${ }^{23}$ While some of this geographical variation may be explained, in part at least, by registration or coding practices, their potential to confound aetiological hypotheses could be reduced (but not overcome entirely) by examining the incidence in one particular region served by a single registry. ${ }^{4}$ Although, overall, there are clear patterns of cancer incidence between the socially disadvantaged and the "well off" sections of the community, the trends for colorectal cancer in the OPCS longitudinal study were not statistically significant. ${ }^{3}$

Just as in numerous reports from England and Wales, ${ }^{5}$ there are significant associations between mortality rates and material deprivation within Northern Ireland. ${ }^{6}$ Thus, the Department of Health and Social Services for Northern Ireland has established strategies to reduce the potential impact on health of material deprivation in the community. ${ }^{7}$ Indeed, the degree of social polarisation in the province may be greater than elsewhere - both ends of the spectrum are inhabited for a number of indicators. We have, for example, a higher proportion of the workforce with no formal qualifications and a higher proportion of school leavers with two or more A levels, the highest birth rate but the lowest proportion of births outside marriage, and the highest proportion of heavy smokers but also the highest proportion of people who abstain from alcohol completely than in any other UK region. ${ }^{8}$

For more than five years Northern Ireland has had a region-wide colorectal cancer register, funded by a local cancer charity. Since very few residents seek care outside the province, this has provided virtually complete data on all patients diagnosed within a stable population of 1.6 million. It therefore provides a valuable resource with which to examine socioeconomic variations in cancer incidence within a well de- 
fined community. Specifically, we have sought to determine whether colorectal cancer incidence or its Dukes's stage distribution differed in areas of high and low material deprivation.

\section{Methods}

The data collated by the Northern Ireland colorectal cancer register for the years 1990 and 1991 form the basis of this report. Information on each new tumour histologically diagnosed in the province is centrally collated. For the study period, this information related to 1178 patients. In addition to pathological data, age and sex are also recorded. A supplementary data-set, based on the clinical information forwarded to the register by the doctor responsible for diagnosing or treating the patient, is also compiled. This second file, from which, for example, tumour site and Dukes's stage could be derived, contained records for 1044 patients.

If the patient's address was not given on either of these records, further attempts were made to abstract it from the hospitals' patient administration systems. The relevant postcode was used to assign the patient's address to one of the 566 electoral wards in the province. Using small area information from the 1991 census, a Townsend deprivation score ${ }^{9}$ was derived for all electoral wards in Northern Ireland. In this manner, the areas from which patients came were characterised according to their degree of affluence/deprivation. The score is calculated as the sum of four normalised and equally weighted census variables (which were originally chosen explicitly to act as proxies for various aspects of a lack of control over material resources). These variables are the percentages of the ward population who are: unemployed, have no car, live in overcrowded housing, and who are not owner occupiers. The resultant scores (ranging from -5.63 to 11.07 ) were ranked and the 566 wards were then grouped into quintiles based on this distribution.

Age standardised incidence rates were calculated for each quintile, standardised to the world standard population. This was first performed for all cases (colon and rectum combined) using the data from the "pathology" file. Poisson regression was used to compare the incidence in each quintile of the population (which characterised the level of deprivation) after first entering terms separately for age and sex. This procedure was then repeated using the data from the "clinical" file. It allowed us to assess the extent of bias that missing data might have caused, and also to analyse colonic and rectal disease separately.

$\chi^{2}$ tests for contingency tables were used to test whether the distribution of Dukes's stage at diagnosis differed according to quintile.

\section{Results}

Overall, it was possible to assign an electoral ward code to 1144 of the patient records in the pathology file $(97 \%)$ and to 1022 of the clinical records $(98 \%$ of the clinical file but $87 \%$ of the overall total in the pathology file). Age standardised incidence rates for colorectal can-
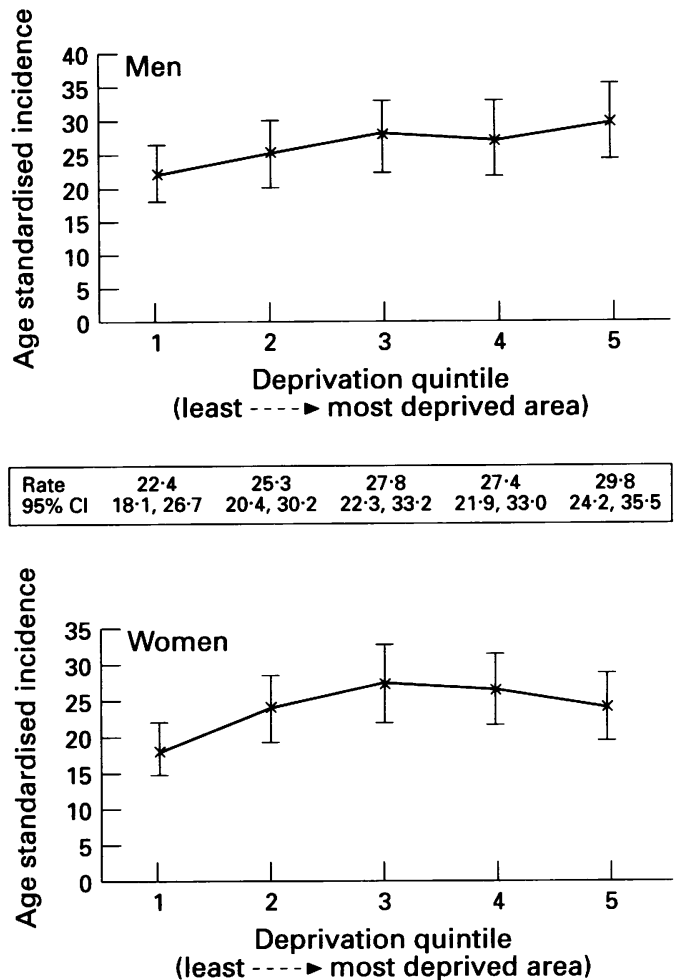

$\begin{array}{lccccc}\text { Rate } & 18 \cdot 4 & 23 \cdot 8 & 27 \cdot 3 & 26 \cdot 5 & 23 \cdot 8 \\ 95 \% \text { Cl } & 14 \cdot 8,21 \cdot 9 & 19 \cdot 2,28 \cdot 4 & 22 \cdot 0,32 \cdot 6 & 21 \cdot 5,31 \cdot 4 & 19 \cdot 3,28 \cdot 4\end{array}$

Figure 1 The directly age standardised colorectal cancer incidence ( $95 \%$ confidence intervals) in men $(n=535)$ and women $(n=609)$ in relation to deprivation quintile in Northern Ireland, 1990-91. (The incidence is $/ 100000$, standardised to the world standard population.)

cer (based on the data from the pathology file) are shown for each quintile in figure 1 . It can be seen that there is an apparent trend in incidence, the lowest being in the well off areas and the highest in the deprived areas.

Table 1 Poisson regression modelling of the incidence (95\% confidence interval) of colorectal cancer based on data from "pathology" and "clinical" registrations in relation to the "deprivation" of the area of residence. Northern Ireland, 1990-91

Deprivation quintile $\beta$ coefficient $\quad$ Rate ratio $\quad(95 \% \mathrm{CI})$ Pathology registrations:$$
\begin{aligned}
& 1 \\
& 2 \\
& 3 \\
& 4 \\
& 5 \\
& + \\
& 1 \\
& + \\
& 1 \\
& 2 \\
& 3 \\
& 4 \\
& 5 \\
& \$ \\
& 1 \\
& 0
\end{aligned}
$$

\begin{tabular}{llll}
1 & & 1 & \\
2 (versus 1) & 0.151 & 1.16 & $(0.97,1.40)$ \\
3 (versus 1) & 0.256 & 1.29 & $(1.08,1.55)$ \\
4 (versus 1) & 0.30 & 1.34 & $(1.12,1.61)$ \\
5 (versus 1) & 0.245 & 1.28 & $(1.06,1.53)$ \\
$*$ Linear trend & 0.063 & 1.06 & $(1.02,1.11)$ \\
+ & & & \\
Clinical registrations: & & & \\
1 & & & \\
2 (versus 1) & 0.180 & 1.20 & $(0.98,1.46)$ \\
3 (versus 1) & 0.246 & 1.28 & $(1.05,1.56)$ \\
4 (versus 1) & 0.295 & 1.34 & $(1.10,1.64)$ \\
5 (versus 1) & 0.238 & 1.27 & $(1.04,1.55)$ \\
$\begin{array}{l}\ddagger \\
\text { Linear trend }\end{array}$ & 0.059 & 1.06 & $(1.02,1.11)$ \\
$\Phi$ & & & \\
\hline
\end{tabular}

* Likelihood ratio statistic, on four degrees of freedom $=13.23$ $p=0.01$ (adjusted for sex and age in five groups: $0-49 \mathrm{y}, 50-59 \mathrm{y}$, $60-69 \mathrm{y}, 70-70 \mathrm{y}$, and $80+\mathrm{y}$ ).

p $69 \mathrm{y}, 70-70 \mathrm{y}$, and $80+\mathrm{y}$ ). $\dagger$ Likelihood ratio statistic, on one degree of freedom $=9.58$ $p=0.002$ (adjusted for sex and age in five groups as specified $\ddagger$ Likelihood ratio statistic, on four degrees of freedom $=10.7$; $\neq$ Likelihood ratio statistic, on four degrees of freedom $=10.7$; $\mathrm{p}=0.03$ (adjusted for sex and age in five groups as specified above).

$\S$ Likelihood ratio statistic, on one degree of freedom $=7.2 ; \mathrm{p}=$ 0.007 (adjusted for sex and age in five groups as specified above). 
Table 2 Poisson regression modelling of colon and rectal cancer incidence (95\% confidence interval) based on data from clinical registration, in relation to "deprivation" of the area of residence. Northern Ireland, 1990-91

\begin{tabular}{lllll}
\hline $\begin{array}{l}\text { Deprivation quintile }- \\
\text { test for linear trend }\end{array}$ & $\begin{array}{l}\text { Rate } \\
\text { ratio }\end{array}$ & $(95 \%$ CI $)$ & $\begin{array}{l}\text { Likelihood } \\
\text { ratio statistic }\end{array}$ & $p$ \\
\hline Colon & 1.05 & $(0.99,1.10)$ & 2.77 & 0.096 \\
Rectum & 1.09 & $(1.01,1.18)$ & 5.24 & 0.022 \\
\hline
\end{tabular}

Table 3 Relationship between Dukes's stage at diagnosis and area of residence in cases of colorectal cancer in Northern Ireland, 1990-91

\begin{tabular}{llll}
\hline Deprivation quintile & Stage $A$ E B & Stage $C$ E D & All \\
\hline Quintiles 1 \& 2 (affluent) & $188(48.6 \%)$ & $199(51.4 \%)$ & $387(100 \%)$ \\
Quintiles 3, 4 \& 5 (deprived) & $265(46.8 \%)$ & $301(53.2 \%)$ & $566(100 \%)$ \\
All & $453(47.5 \%)$ & $500(52.5 \%)$ & $953(100 \%)$ \\
\hline
\end{tabular}

$\chi^{2}$ statistic $=0.22 ; \mathrm{df}=1 ; \mathrm{p}=0.64$

${ }^{*}$ For 91 cases, neither stage nor quintile could be determined.

This trend was tested formally by Poisson regression, the results of which are shown in table 1 , which gives the rate ratios and $(95 \%$ confidence intervals) for each quintile. (Because of small numbers of cases in the very young age groups, the $0-19,20-29,30-39$, and 40-49 year-old age groups have been collapsed into one category). The $\chi^{2}$ likelihood-ratio statistic for a linear trend term for deprivation was significant at $p=0.002$, and indicated that moving between consecutive quintiles resulted in a $6 \%$ increase in incidence $(95 \%$ CI $2 \%$, $11 \%)$. Table 1 also shows the same analysis for data from the clinical file and indicates an effect of identical size. (The fit of the model was satisfactory - the final deviance was equal to 43.7 on 39 degrees of freedom). There was no statistical interaction between the deprivation effect and age group (data not shown). Although the trend shown in figure 1 tends to plateau and is not so marked for women, there was no statistical interaction between the deprivation effect and gender $\left(\chi^{2}=1.45, \mathrm{df}=1\right.$, $\mathrm{p}=0.23$ ).

Table 2 gives the results of the analysis of colon and rectum cases separately. As can be seen, the deprivation effect is more apparent for rectal cases, producing a significant $9 \%$ change in the incidence for each unit change in deprivation (that is, moving from one quintile to the next) compared with $5 \%$ for colon cancer.

Table 3 illustrates the Dukes's stage at diagnosis for cases for whom both stage and electoral ward of residence could be established. There was no statistically significant difference in the proportion of early and advanced stage tumours between patients from the relatively affluent and those from the deprived areas.

\section{Discussion}

Our findings suggest that socioeconomic conditions, if not causal, are at least associated with factors determining the occurrence of colorectal cancer in the Northern Ireland population. Numerous aetiological factors have been previously investigated, including a sedentary lifestyle,${ }^{10}$ body mass index, ${ }^{11}$ energy intake, ${ }^{12}$ habitual alcohol or coffee consumption ${ }^{13}$ and, of course, diet. ${ }^{14}$ In fact, while many of these are known to aggregate with indicators of social disadvantage, the evidence linking the latter with bowel cancer risk, especially in previous British studies, has not been conclusive.

For instance, in the OPCS longitudinal study, the standardised incidence ratio (SIR) for colon cancer was 93 in council tenants but 106 for those in privately rented accommodation. ${ }^{15}$ While the SIR for the manual social classes was 100 , it was 95 for those in non-manual classes. Neither these differences nor those for rectal cancer (which were in the opposite direction) were statistically significant. ${ }^{14}$ Earlier analyses of the relationship between car ownership and incidence similarly showed no statistically significant trend. ${ }^{3}$ Despite inconclusive evidence from previous British studies, a recent study from Italy found a lower colorectal cancer incidence in manual workers and in subjects with no more than primary school education. The authors explained this association by virtue of the lower social classes in Italy having a healthier diet that is lower in fat and higher in fibre. ${ }^{16} \mathrm{~A}$ converse argument could be used to explain the present findings, however, as a relatively "unhealthy" diet, low in fruit and vegetables, is known to be more common among the materially disadvantaged in Northern Ireland. ${ }^{17}$ Though a common dietary hypothesis might explain the opposite social class patterns of incidence in the OPCS longitudinal study and this recent Italian study, it would be insufficient to explain the fact that both of these studies found appreciably raised rates among the unemployed, and cannot account for much of the recent trend in incidence and subset distribution of the disease. ${ }^{1819}$

It is not possible to contrast directly our results with those from Scotland. ${ }^{20}$ The latter demonstrated a higher incidence in the least deprived fifth of the population but the analyses were for all colon and rectal cases combined. Whether the trends for colonic and rectal disease were the same in Scotland is not known. Our own results suggest that different patterns of socially determined risk factors influence colon and rectal cancer incidence. Results from other studies are somewhat inconsistent. In Sweden, colon but not rectal cancer was associated with white collar occupations, ${ }^{21}$ while in Finland both colon and rectal cancer were associated with higher socioeconomic status and higher levels of education. ${ }^{22}$ In Italy, on the other hand, education was inversely associated with rectal cancer incidence and positively associated with colon cancer. ${ }^{23}$ Though probably correlated with a community's social class structure and with education, the formulation of the Townsend score is intended to reflect relative command over resources and it is possible that apparently disparate results might arise because subtly different aspects of a "deprivation" effect (whatever that might be) are being measured in different ways in different studies. For instance, in the Whitehall study, employment grade and car ownership contributed independently to all cause mortality. ${ }^{24}$ We elected to utilise the Townsend deprivation score as local work had already demonstrated 
its association with other population health indicators. $^{6}$

Even though the molecular origin of bowel cancer is increasingly understood, ${ }^{25}$ the fact that there is a significant trend in incidence associated with material deprivation suggests that a proportion may be preventable. That this trend was more noticeable for rectal cancer might offer suggestions for future aetiological research. Some risk factors for rectal cancer, such as beer drinking, ${ }^{26}$ seem to be distinct from those for colon cancer and could possibly account for some of the socioeconomic variation. It is obviously possible, if not likely, that genetic factors, such as acetylator status, might modify the risk associated with "socially" determined dietary factors. ${ }^{27}$ Depending on the magnitude of their effects and the dietary variation across social strata, it may be unnecessary to posit that the genetic factors themselves are more or less prevalent in any given social stratum. For example, other risk factors such as smoking, which may be associated with social class or deprivation, may modify the risks attributable to acetylator status. ${ }^{27}$

Very few of the cited studies have examined the extent of social variation in colorectal cancer incidence after adjusting for other known risk factors. However, a recent study from The Netherlands showed a socioeconomic differential in colon cancer incidence even after adjusting for other dietary and non-dietary risk factors. ${ }^{28}$ Thus, whether it is possible to devise social strategies to reduce the socioeconomic variation in colorectal cancer incidence is open to question. Since cancer has a long induction period it is not clear whether the associations observed reflect the influence of socioeconomic conditions on the disease process in adult or in early life. Many people move house several times during their lives and to separate properly the cohort from period effects ${ }^{29}$ would require a prospective study.

Our data suggest that there was little difference in the frequency of more advanced tumours in patients from the deprived and the well off areas. Pickering et al have described significant variation in survival from bowel cancer across Wessex according to the district of residence but, unfortunately, they had no information on stage at diagnosis. ${ }^{30}$ Recently, Riley et al reported that HMO enrollees in the USA were more likely to have colon cancer diagnosed at an early stage and they attributed their findings to better access to or usage of preventive health care.$^{31}$ However, faecal occult blood screening is not routinely offered in Northern Ireland and the province's population has a higher per capita number of doctors and hospital beds than most other regions in the UK. ${ }^{7}$ Although our findings only refer to data on clinically registered cases, we have no reason to believe that missing data for postcode and tumour stage were anything other than nondifferentially related. We have found that clinical registration is more a reflection of the practice of individual surgeons rather than any given area $^{32}$ and none of the surgeons serve patients from exclusively well off or deprived areas. Further data collation for later years may shed more light on this issue.

Our conclusions must be tempered by a number of caveats. The sort of analysis used in this study is obviously susceptible to the "ecological fallacy" if it turned out that relatively well off patients from the poor areas were the ones being diagnosed with colorectal cancer. Nevertheless, the method has been considered to be relatively robust ${ }^{33}$ and significant associations between measures of material deprivation and ill health have been demonstrated both at area and individual levels. ${ }^{34-36}$

Though our ability to assign a ward code to the patient records compares favourably with the completeness of other similar studies, the possibility exists that its absence for around $10 \%$ of records may have biased our sample. The fact that the results obtained for both sites combined using the more complete data from the "pathology" file were comparable to those obtained using the less complete "clinical" file suggests that our conclusions are probably insensitive to the incompleteness of postcoded address data, at least over this range. One of the strengths of the OPCS longitudinal study was the relative completeness of baseline and follow up data but, by comparison with our own data-set, their analyses had less power to confirm the putative associations.

We feel that our conclusions are made the more robust by the register's high level of case ascertainment. Although we may have missed a small number of cases (predominantly, we surmise, among the very old) that were not histologically diagnosed, there was no interaction between the deprivation effect and age and exlcusion of the very old cases ( $>80$ years) would not have altered our conclusions. Crude measures of the reliability of registry data include the ratio of deaths to registrations and the ratio of histological diagnoses to registrations. ${ }^{37}$ During 1990/91 there were 876 colorectal cancer deaths in Northern Ireland. The ratio 876/ $1178(0.74)$ is very close to that for England and Wales as a whole (0.73) and is similar to that reported for Northern Ireland in $1990 .^{33} 38$ Although the Department of Health has now made a considerable investment in the Northern Ireland cancer register (that is, the government-funded register for all tumours), five years ago the reported ratio of histological diagnoses to registrations for bowel cancer were 0.86. Although this ratio is often used for international comparisons, it is a rather crude indicator, for unless multiple data sources are available, the incidence date will usually not be known for cases registered by death certificate only. All of the cases in our register were histologically diagnosed. Cases that might otherwise have been ascertained by death certificate only were not included but since our coverage of histologically diagnosed cases was complete we consider that few cases that were incident in 1990-91 were missed.

We gratefully acknowledge the help of all the surgeons and pathologists who have contributed to the register. The Northern Ireland colorectal cancer register is supported by The Friends of Montgomery House.

We appreciate the assistance of Ms Penny McDonald for help in tracing unknown postcodes and in preparing the figures. 
1 Parkin DM, Muir CS, Whelan SL, Gao YT, Ferlay J, Powell $\mathrm{J}$ eds. Cancer incidence in five continents, Vol VI. Lyon: World Health Organisation, International Agency for Research on Cancer, 1992.

2 Swerdlow A, dos Santa Silva I. Cancer Research Campaign: Atlas of cancer incidence in England and Wales. Oxford: Atlas of cancer incidence in Engla

3 Leon DA. Longitudinal study. Social distribution of cancer. London: OPCS Series LS (3), 1988

4 Swerdlow AJ. Cancer registration in England and Wales: Some aspects relevant to interpretation of the data. $\mathcal{F} R$ Stat Soc 1986;149:146-60.

5 Smith GD, Bartley M, Blane D. The Black Report on socioeconomic inequalities in health 10 years on. $B M F$ 1990;301:373-7

6 Policy Research Institute. Spatial and social variations in the distribution of health indicators in Northern Ireland. Belfast: Policy Research Institute, Queen's University, 1990.

7 Department of Health and Social Services (NI). A framework for health and personal social services in Northern Ireland, 1992-97. Belfast: Department of Health and Social Services (NI), 1992

8 Central Statistical Office. Social trends 30, 1995. London: Central Statistical Office, 1995.

9 Townsend P, Phillimore P, Beattie A. Health and deprivation inequality and the north. London: Croom Helm, 1988.

10 Bartram HP, Wynder EL. Physical activity and colon cance risk? Physiological considerations. Am f Gastroenterol 1989; 84:109-12.

11 Suadicani P, Hein HO, Gyntelberg F. Height, weight and risk of colorectal cancer. An 18 year follow-up in a cohort of 5249 men. Scan 7 Gastroenerol 1993;28:285-88.

12 Little J, Logan RFA, Hawtin PG, Hardcastle JD, Turner ID. Colorectal adenomas and energy intake, body size and physical activity: a case-control study of subjects and physical activity: a case-control study of subjects participating in the Nottingham faecal occult

13 Williams RR, Horm JW. Association of cancer sites with tobacco and alcohol consumption and the socio-economic status of patients: interview from the third national cancer survey. F Natl Cancer Inst 1977;58:?? ??

14 Tricholpoulus D, Polychronupulous A. Epidemiology, die and colorectal cancer. Eur $\mathcal{f}$ Cancer Clin Oncol 1986;22 335-7.

15 Kogevinas E. Longitudinal study 1971-83: socio-demographic differences in cancer survival. London: OPCS, Series LS (5), 1989.

16 Faggiano F, Zanetti R, Costa G. Cancer risk and social inequalities in Italy. $\mathcal{F}$ Epidemiol Community Health 1994; 48:447-52.

17 McClean R, McCrum EE, Scally G, et al. Dietary patterns in the Belfast MONICA project. Proc Nutr Society 1990; 49:297-305.

18 Kee F, Collins BJ, Patterson CC. Trends in the incidence of colo-rectal cancer in the north and south of Ireland 1950-1989. F Epidemiol Community Health 1990;44:??-??

19 Kee F, Wilson RH, Gilliland R, Sloan JM, Rowlands B Moorehead J. Changing site distribution of colo-recta cancer. BMF 1992;305:158.
20 Sharp L, Black R, Harkness E, Finlayson A, Muir C. Cancer registration statisics Scotland 1981-1990. Edinburgh: Scottish Cancer Intelligence Unit, ISD, 1993.

21 Vagero D, Persson G. Occurrence of cancer in socio-economic groups in Sweden. Scan F Soc Med 1986;14:151-60.

22 Pukkala E, Teppo L. Socio-economic status and education as risk determinants of gastrointestinal cancer. Prev Med 1986;15:127-38

23 Ferraroni M, Negri E, La Vecchia C, D’Avanzo B, Franceschi S. Socio-economic indicators, tobacco and alcohol in the aetiology of digestive tract neoplasms. Int $\mathcal{F}$ Epidemiol 1989;18:556-62.

24 Smith GD, Shipley MJ, Rose G. Magnitude and causes of socio-economic differentials in mortality: further evidence from the Whitehall study. 7 Epidemiol Community Health $1990 ; 44: 265-70$

25 McMichael AJ. Molecular epidemiology: new pathway or new travelling companion? Am $\mathcal{F}$ Epidemiol 1994;140:1-11.

26 Kune S, Kune GA, Watson LF. Case-control study of alcoholic as aetiological factors: the Melbourne colorectal cancer study. Nutr Cancer 1987;9:43-7.

27 Probst-Hensch N, Haile RW, Ingles S, et al. Acetylation polymorphism and prevalence of colorectal adenomas. pancer $1995 ; 55: 2017-20$.

28 van Loon AJM, van den Brandt P, Golbohm RA. Socioeconomic status and colon cancer incidence: a prospective cohort study. Br ₹ Cancer 1995;71:882-7.

29 Kee F, Collins B, Patterson CC. Colorectal cancer in the north and south of Ireland 1950-1984. F Epidemiol Community Health 1990;44:220-3.

30 Pickering RM, Chadwell I, Mountney L. Importance of district of residence and known primary site for bowel cancer survival: analysis of data from Wessex cancer registry. $\mathcal{F}$ Epidemiol Community Health 1992;46:266-70.

31 Riley GF, Potosky A, Luitz JD, Brown M. Stage of cancer at diagnosis for Medicare HMO and fee-for-service enrollees. diagnosis for Medicare HMO and fee-for-

32 Wilson R, Moorehead RJ. Preliminary findings of the Northerm Ireland colorectal cancer register 1990-91. Belfast: NICCR 1992.

33 Kreiger N. Overcoming the absence of socioeconomic data in medical records: validation and application of a censusbased methodology. Am $\mathcal{F}$ Public Health 1992;92:703-10.

34 Policy Research Institute. Spatial and social variations in the distribution of health indicators in Northern Ireland. Belfast: Policy Research Institute, Queen's University, 1990.

35 Curtis SE. Use of survey data and small area statistics to assess the link between individual morbidity and neighbourhood deprivation. 7 Epidemiol Community Health 1990;44:62-8.

36 Mays N, Chinn S. Relation between all cause standardised mortality ratios and two indices of deprivation at regional 1989;43:191-99.

37 Cancer Research Campaign. Factsheet 1.3. London: CRC 1987

38 Kee F, Collins BJ, Patterson CC. Incidence and site distribution of colo-rectal cancer in Northern Ireland. Ulster Med f 1990;59:155-60. 